\title{
Kangaroo mother care in resource-limited settings: implementation, health benefits, and cost-effectiveness
}

This article was published in the following Dove Press journal:

Research and Reports in Neonatology

30 June 2017

Number of times this article has been viewed

\section{Samuel N Uwaezuoke \\ Department of Pediatrics, University of Nigeria Teaching Hospital, Ituku- Ozalla, Enugu, Nigeria}

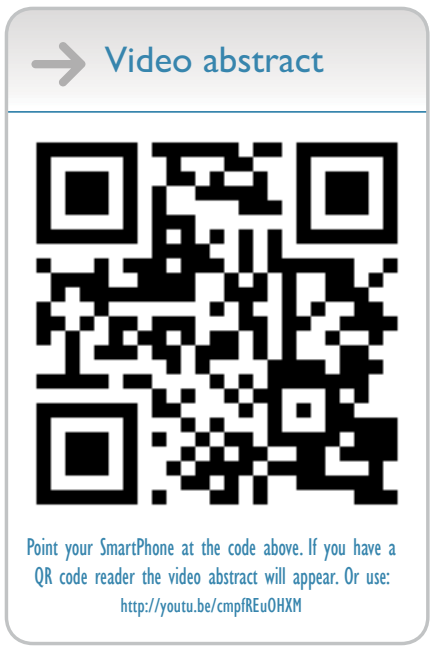

Correspondence: Samuel N Uwaezuoke Department of Pediatrics, University of Nigeria Teaching Hospital, Ituku-Ozalla, PMB 014429, Enugu, 40000I, Nigeria Tel +2348033248I08 Email snuwaezuoke@yahoo.com

\begin{abstract}
Kangaroo mother care (KMC) represents an intervention in low birth weight infants for resource-limited settings which aims to reduce mortality rates by thermoregulation, supporting breastfeeding, and promoting early hospital discharge. In terms of cost and impact on neonatal survival, it has comparative advantages over the conventional method of care (CMC). This paper aimed to review the evidence concerning the progress of KMC implementation, its health benefits, and its cost-effectiveness, especially in developing countries. From the synthesized evidence, $\mathrm{KMC}$ was shown to be a useful adjunct to CMC particularly with respect to improving neonatal survival, supporting breastfeeding, and promoting early discharge from the hospital. Substantial progress has been made in its implementation in many developing countries where facility-based KMC has been institutionalized. Despite the cost-effectiveness of KMC in neonatal care, its global implementation is bedeviled with country-specific, multifaceted challenges. In developed countries, there is an implementation gap due to easy accessibility to technologybased CMC. Nevertheless, many developing countries have initiated national policies to scale up $\mathrm{KMC}$ services in their domain. Given the major constraints to program implementation peculiar to these resource-limited countries, it has become imperative to boost caregiver confidence and experience using dedicated spaces in the hospital, as well as dedicated staff meant for adequate ambulatory follow-up and continuous health education. Capacity training for health professionals and provision of space infrastructure thus constitute the basic needs which could be funded by International Aid Agencies in order to scale up the program in these settings.
\end{abstract}

Keywords: neonatal care, low birth weight infants, thermoregulation, breastfeeding, neonatal survival, developing countries

\section{Introduction}

Kangaroo mother care (KMC) has long been recommended and used as the "natural" means of thermoregulation for preterm and low birth weight (LBW) infants, in both the developed and developing world. ${ }^{1}$ Thermoregulation is the ability to maintain a balance between thermogenesis (heat production) and thermal loss (heat loss) in order to regulate body temperature within a specific normal range. ${ }^{2}$ In the newborn infant, thermoregulation is a critical physiologic function which is strongly affected by physical immaturity, disease severity, and environmental factors. ${ }^{3}$ Physiologically, the neonate is susceptible to cold stress because of the following factors: higher ratio of body surface area (promotes thermal loss) to body volume (proportional to thermogenesis), paucity of thermal insulation from subcutaneous fat, poor muscle bulk and inability to shiver, as well as underdeveloped nervous system with poor response to cold. ${ }^{3}$ Cold stress (hypothermia) portends grave metabolic consequences for all neonates: especially the 
preterm and LBW babies who are more vulnerable to these consequences, leading to increased morbidity and mortality rates. Thus, preventing hypothermia is vital to neonatal survival and long-term outcome.

To prevent hypothermia, the newborn infant is well endowed with brown adipose tissue or brown fat which basically plays a vital role in thermoregulation; ${ }^{4}$ it generates heat by non-shivering thermogenesis and helps to distribute heat throughout the body because of its abundant vascularization. ${ }^{5}$ Thus, thermogenesis in brown adipose tissue provides the neonate with an alternative means of thermoregulation.

Given the susceptibility of newborn infants to temperature instability, nursing them within their thermoneutral environment is essential in order to appropriately manage and ameliorate the effects of cold stress (hypothermia). The thermoneutral environment refers to the environmental air temperature at which an infant with a normal body temperature has a minimal metabolic rate and therefore minimal oxygen consumption. ${ }^{6}$

As a fundamental component of neonatal care, the maintenance of the thermoneutral environment is therefore the ultimate goal of neonatal temperature control and management. Since the three major contributors to perinatal mortality globally (namely prematurity, birth asphyxia, and congenital anomalies) are associated with increased risk of hypothermia, thermoregulation remains a veritable tool in reducing perinatal mortality rates (PNMRs). Optimum thermoregulation and related nursing care clearly revolve around the methods of thermometry, the choice of environment, and the interventions for temperature instability.

One of the major strategies for reducing PNMRs is the establishment of neonatal intensive care units (NICUs) equipped with cutting-edge technology for neonatal care. Such units basically require both adequate staffing and adequate equipment such as incubators, ventilators, phototherapy units, blood pressure monitors, and oxygen hood.

In developed countries, the technology-driven conventional method of care (CMC) is categorized into "levels of care," with the more advanced level reserved for the critically ill and vulnerable newborn infants. At each level of care, thermoregulation remains an essential component, as modern incubators (representing the choice of environment and intervention for temperature instability) are readily available for this purpose. Other components of CMC include cardiopulmonary monitors, oximetry, intravenous infusions, and continuous positive airway pressure ventilators. In contrast, there is a dearth of such equipment in resource-limited developing countries; in these settings, reports show that PNMRs have remained unacceptably high in comparison with the figures from the developed world. ${ }^{7,8}$

To address this disparity in mortality statistics, attention has now been directed at adopting KMC as an adjunct and not necessarily an alternative method to CMC for LBW infants in resource-limited countries. KMC has gained global acceptance with several studies showing that it can lead to improvement in neonatal survival and outcome, especially in LBW infants. ${ }^{9-12}$

This paper aimed to review the evidence concerning the progress of KMC implementation, its health benefits, and its cost-effectiveness in developing countries.

\section{KMC: historical perspective and progress of implementation}

KMC was first initiated in 1978 in Bogotá (Colombia) following the challenges of overcrowding and limited resources in NICU which resulted in high morbidity and mortality among LBW infants; the intervention comprised continuous skinto-skin contact between the mother and the infant, exclusive breastfeeding, and promoting early home discharge in the kangaroo position accompanied with follow-up. ${ }^{13}$

In an early Cochrane systematic review conducted on the impact of KMC on morbidity and mortality in LBW infants, the authors concluded that although KMC appeared to minimize severe infant morbidity without any reported harmful effect, there was no ample evidence yet to recommend its routine use in LBW infants. ${ }^{14}$ Evidence from subsequent updated reviews by these authors supports the use of KMC in LBW infants as both as an adjunct and a substitute to CMC particularly in resource-limited settings. ${ }^{15-17}$ However, the noted gray areas include the efficacy and safety of early onset continuous KMC in unstabilized LBW infants, the long-term neurodevelopmental outcomes, and the costs of care ${ }^{17}$ Despite these issues which need further evaluation, $\mathrm{KMC}$ is inexorably linked to infant feeding, and thus exerts an influence on long-term breastfeeding.

To underscore this role in promoting breastfeeding, a study in Sweden has reported that very preterm infants who breastfed at 1, 2, 5, and 6 months were those who spent more time in KMC per day than those not breastfeeding at these periods. ${ }^{18}$ In their work, the authors aimed to investigate the use of $\mathrm{KMC}$ and its relationship with breastfeeding at 1-6 months of corrected age in mothers of very preterm and preterm singleton infants. In contrast, there were no statistically significant differences among the preterm infants with respect to the amount of KMC per day between the breastfed infants and the non-breastfed infants, making the authors 
conclude that KMC has a positive impact on the process of breastfeeding, especially in the very preterm infants who are more vulnerable and have to be exposed to longer duration of KMC. ${ }^{18}$ In other words, the duration of KMC determines the extent to which infant-maternal bonding is fostered, which in turn encourages the act of breastfeeding.

Recently, some developing countries have evaluated the progress in the implementation of KMC and adopted programs which targeted the scaling up of this method of neonatal care. For example, a multi-country, cross-sectional, and mixed-design study systematically evaluated the implementation status of facility-based KMC services in four African countries: Mali, Malawi, Uganda, and Rwanda. ${ }^{19}$ Across these four countries, 95\% of evaluated health facilities demonstrated some evidence of KMC practice. Health institutions that fared better had a longer history of KMC implementation or had been structured as centers of excellence or had strong leadership engaged in promoting the implementation process, ${ }^{19}$ the authors thus advocated that the integration of KMC into routine newborn care services should be a component of all maternal and newborn care programs.

Similarly, a study in Ghana (in the West African subregion), which aimed to assess the progress with the implementation of KMC for LBW infants at four regions, reported that $68 \%$ of the surveyed hospitals demonstrated sufficient progress with its implementation. ${ }^{20} \mathrm{KMC}$ implementation involved the incorporation of care into existing conventional neonatal care services. Specifically, $50 \%$ of these hospitals had set aside a special ward for KMC, while $66 \%$ of them employed a record system dedicated for infants receiving KMC. Obviously, a modest progress has been made in this country but providing adequate maternal support and followup services were suggested as measures for the successful implementation of $\mathrm{KMC} .{ }^{20}$

A case study in Asia was also conducted to appreciate the institutionalization processes of facility-based KMC services in three Asian countries (India, Indonesia, and the Philippines), as well as to identify the factors responsible for the slow uptake of KMC in these countries. ${ }^{21}$ The investigators noted that the establishment of KMC services at individual health facilities commenced several years prior to the official prioritization for scale up. They also identified three major themes in the institutionalization of $\mathrm{KMC}$ : pioneers of facility-based $\mathrm{KMC}$; patterns of KMC knowledge and skills dissemination; and uptake and expansion of KMC services based on global trends and national policies. ${ }^{22}$
In the experience of these countries, pioneers of facilitybased KMC firstly were acquainted with the concept in the 1990s and instituted the practice in a few individual tertiary health facilities, without further dissemination. Secondly, a training method helpful to the early establishment of KMC services was to sponsor institutional health professional teams to acquire more knowledge outside these countries, especially in Colombia. Thirdly, further national step-down subsequently occurred and was followed by integration of $\mathrm{KMC}$ into neonatal and obstetric care programs. ${ }^{21}$ The intermittent uptake and expansion of $\mathrm{KMC}$ services occurred in three phases which conformed to contemporary global trends: the pioneer phase with individual champions while the worldwide attention was on child survival (1998-2006); the newborn-care phase (2007-2012); and lastly the prevalent phase where very LBW newborn infants are also included in action plans. ${ }^{21}$

Based on the World Health Organization's (WHO) approval of KMC for stabilized newborn infants in health facilities located in both high-income and low-resource settings, a group of researchers set out to utilize a 12-country analysis to explore health system bottlenecks affecting the scale up of $\mathrm{KMC}$, to proffer solutions to the most significant bottlenecks, and to outline priority actions for scale up. ${ }^{22}$ The bottleneck analysis tool was applied in 12 countries in Africa and Asia as part of the Every Newborn Action Plan process. Their findings included remarkable differences in the perceived severity of health system bottlenecks between Asian and African countries, with the former reporting more significant bottlenecks for KMC with respect to all the health system building blocks. ${ }^{22}$ The significant bottlenecks for KMC were found in community ownership and health financing (especially in South Asia), leadership and governance, as well as health workforce building blocks. The authors in conclusion identified and suggested a combination of three pathways for a more rapid scale up of KMC: champion-led, project-initiated, and health systems designed pathways. ${ }^{22}$

In another study conducted to identify factors responsible for the unsuccessful implementation of KMC in 15 developing countries, the authors reported the early discharge component (including ambulatory follow-up) as the most difficult to implement. ${ }^{23}$

Notably, resistance from health professionals, mothers, and families was frequently related to local cultural practices. ${ }^{23}$ Thus, caregiver confidence and experience now appear to be the major constraint to KMC implementation in developing settings. 


\section{Health benefits of KMC in resource- limited settings}

Generally, KMC has multipronged benefits for the parents, preterm and LBW infants, health institutions, and the community. For the parents, KMC promotes parent-child attachment and bonding, improves parental confidence as caregivers, and promotes infant nutrition..$^{17,24,25}$ For the preterm and LBW infants receiving KMC, they experience more normalized vital signs (temperature, heart rate, respiratory rate, and oxygen saturation), ${ }^{26-28}$ increased weight gain, ${ }^{17,28}$ and fewer hospital-acquired or nosocomial infections. ${ }^{17}$ Other reported health benefits include improved cognitive development, normalized growth, reduced pain responses, and positive effects on motor development, ${ }^{28-30}$ as well as improvement of sleep patterns and amelioration of colic. ${ }^{31}$ Another report also suggests that KMC may facilitate oral growth and development in preterm infants. ${ }^{32}$

Expectedly, for health institutions, KMC results in reduced need for the more expensive CMC and increased parental involvement and opportunities for health education, while the larger community stands to benefit from reduction in hospital-associated costs.

As previously mentioned, evidence from a recent systematic review indeed supports the use of KMC as a possible substitute for CMC in settings where resources are constrained. ${ }^{17}$ Nevertheless, other researchers (who studied the provision of neonatal care for premature infants at a district level within a resource-limited setting in Burundi, Central Africa) reported high survival rates in the absence of high-tech equipment or specialist health care personnel. ${ }^{33}$
They suggested that these results were achieved through the provision of complementary NICU and KMC units among other proffered factors. Thus, it is reasonable to advocate for an effective NICU using low technology with an effective complementary KMC in resource-limited countries.

Specifically, studies in different parts of the developing world have clearly shown these health benefits for LBW infants to include better survival outcome, increased growth parameters, and reduced duration of hospitalization as well as sustenance of exclusive breastfeeding (Table 1). For example, in southern Africa, a study which was conducted in a Mozambican hospital without facilities for intensive care, revealed that $\mathrm{KMC}$ was a feasible and appropriate method of care. ${ }^{34}$ The authors observed that out of 32 hospitalized LBW infants weighing $\leq 1.8 \mathrm{~kg}$, survival was $73 \%$ in $22 \mathrm{KMC}$ and $20 \%$ in 10 non-KMC infants. ${ }^{34}$ The findings of this study underscore the role of $\mathrm{KMC}$ in improving the mortality and morbidity outcomes in LBW infants. Furthermore, the findings of the Cochrane review which synthesized data from 21 studies comprising 3,042 LBW infants weighing $\leq 1.5 \mathrm{~kg}$ lend credence to this role, as the review reported reduced risk of mortality, hospital-acquired infection, and hypothermia coupled with increased anthropometric parameters and rates of breastfeeding among these infants. ${ }^{17}$ This could be the basis for the inclusion of "preterm infants weighing $\leq 1.5 \mathrm{~kg}$ " and "independent breathing" as the original KMC eligibility criteria for LBW babies.

In India, a randomized control study of 28 stabilized very $\mathrm{LBW}$ neonates weighing $<1.5 \mathrm{~kg}$ was conducted to determine the effect of $\mathrm{KMC}$ on breastfeeding rates, weight

Table I KMC versus CMC: summary of selected study findings

\begin{tabular}{|c|c|c|c|}
\hline Authors (country), year & Some evaluated parameters & KMC group & CMC group \\
\hline \multirow[t]{2}{*}{ Lincetto et al (Mozambique), ${ }^{34} 2000$} & Survival rate (proportion of admitted & $73 \%$ & $20 \%$ \\
\hline & LBW infants that survived) & & \\
\hline \multirow[t]{4}{*}{ Ramanathan et al (India), ${ }^{35} 200 \mathrm{I}$} & Weight gain after first week of life & $15.9 \pm 4.5 \mathrm{~g} /$ day & $10.6 \pm 4.5 \mathrm{~g} /$ day \\
\hline & Hospital discharge & $27.2 \pm 7$ days & $34.6 \pm 7$ days \\
\hline & Number of mothers exclusively & $12 / 14$ & $6 / 14$ \\
\hline & breastfeeding at 6 weeks & & \\
\hline \multirow[t]{4}{*}{ Suman et al (India), ${ }^{36} 2008$} & Average weight gain/day & $23.99 \mathrm{~g}$ & $15.58 \mathrm{~g}$ \\
\hline & Weekly increments in OFC & $0.75 \mathrm{~cm}$ & $0.49 \mathrm{~cm}$ \\
\hline & Weekly increments in length & $0.99 \mathrm{~cm}$ & $0.7 \mathrm{~cm}$ \\
\hline & $\begin{array}{l}\text { Number of exclusively breastfed babies } \\
\text { after study }\end{array}$ & $98 \%$ & $76 \%$ \\
\hline Worku and Kassie (Ethiopia), ${ }^{38} 2005$ & Mortality rate & $22.5 \%$ & $38 \%$ \\
\hline Lima et al (Brazil), ${ }^{39} 2000$ & Cost of care & 20 US\$ per bed/day & 66 US\$ per bed/day \\
\hline \multirow[t]{3}{*}{ Charpak et al (Colombia), ${ }^{40} 200 \mathrm{I}$} & Cumulative mortality at I 2 months & $3.0 \%$ & $5.5 \%$ \\
\hline & Hospital stay $(\leq 1.2 \mathrm{~kg})$ & 6.2 days & 19.7 days \\
\hline & Hospital stay $(\leq 1.5 \mathrm{~kg})$ & 4.0 days & I3.8 days \\
\hline
\end{tabular}

Abbreviations: KMC, kangaroo mother care; CMC, conventional method of care; LBW, low birth weight; OFC, occipitofrontal circumference. 
gain, and duration of hospitalization. ${ }^{35}$ Two groups, the kangaroo group $(n=14)$ and the control group $(n=14)$ were subjected to KMC and CMC, respectively. Remarkably, the neonates in the kangaroo group significantly showed better weight gain after the first week of life and earlier hospital discharge compared to the control group. In addition, the number of mothers who exclusively breastfed their babies at 6-week follow-up was twice more in the kangaroo group than in the control group. Thus, the authors also concluded that the demonstrable effectiveness of $\mathrm{KMC}$ in improving neonatal outcome measures in LBW infants makes it an excellent adjunct to $\mathrm{CMC}$ in a nursery. ${ }^{35}$

In another related study in India, Suman et al compared the effect of KMC and CMC on growth parameters in 206 LBW infants weighing $<2 \mathrm{~kg}$ at birth. ${ }^{36}$ These neonates were randomized into two groups: the intervention group $(n=103)$ who received KMC and the control group $(n=103)$ who received $\mathrm{CMC}$. Babies in the intervention (KMC) group significantly had better average weight gain per day, and higher weekly increments in head circumference and length. Furthermore, a significantly higher proportion of babies in the control (CMC) group experienced hypothermia, hypoglycemia, and sepsis. More KMC babies were exclusively breastfed at the end of the study compared to their CMC counterparts. Obviously, KMC did not only improve growth but was also shown to reduce morbidities in LBW infants. ${ }^{36}$

The ameliorative effect of KMC on neonatal morbidities, such as hypothermia, has been corroborated by other investigators who reported a significant reduction of hypothermia, higher oxygen saturations, and decrease in respiratory rates in neonates who received $\mathrm{KMC}$ versus those who received CMC. ${ }^{37}$

Another randomized controlled trial, which was conducted in Ethiopia, aimed to study the effectiveness of early $\mathrm{KMC}$ before stabilization of LBW infants as compared with the CMC. ${ }^{38}$ The investigators reported remarkable differences in the proportion of babies under both methods of care who were on intravenous fluids ( $58 \%$ of KMC vs $52 \%$ of CMC) and on intranasal oxygen (34\% of KMC vs $37 \%$ of CMC), as well as their mean age at exit from the study (4.6 days for $\mathrm{KMC}$ vs 5.4 days for $\mathrm{CMC}$ ) and their mortality statistics ( $22.5 \%$ of KMC vs $38 \%$ of CMC). Generally, their report indicates that survival for the preterm LBW babies was much better for the early KMC group than for their counterparts in the CMC group within 12 hours and thereafter. ${ }^{38}$

In a descriptive study on KMC of LBW infants conducted in a tertiary care hospital in Brazil, Lima et al documented another evidence regarding the efficacy of this method of care on neonatal survival, and on the promotion of breastfeeding. ${ }^{39}$ Their major findings include absence of recorded mortality in the hospital, absence of episodes of moderate or severe hypothermia, and a daily weight gain of $15 \mathrm{~g}$ during KMC. Moreover, at follow-up, $87 \%$ of the babies were still exclusively breastfed at 1 month and $63 \%$ at 3 months, while the cost of KMC compared to CMC was less (US\$ 20 vs US\$ 66 per bed/day). The authors thus confirmed that KMC for stabilized, hospitalized LBW infants was not only feasible but was also less expensive, and should serve as an appropriate alternative to $\mathrm{CMC}$ in resource-limited settings. ${ }^{39}$

In Colombia, another randomized controlled trial to assess the effectiveness and safety of KMC for LBW infants revealed that the mortality risk was lower among infants who received this method of care, although the difference was not statistically significant (KMC: 11 [3.1\%] of 339; control infants: 19 [5.5\%] of 324 ; relative risk: $0.57 ; 95 \%$ confidence interval [CI]: $0.17-1.18) .{ }^{40}$ Furthermore, the growth index of occipitofrontal circumference was significantly greater in the KMC group, but the developmental indices of the two groups were similar. Infants who weighed $\leq 1.5 \mathrm{~kg}$ at birth and received KMC had shorter duration of admission than those who received CMC. The number of infections was similar in the two groups, but the severity was less among infants who received KMC. More of these infants were breastfed until 3 months of corrected age. ${ }^{40}$

\section{The cost-effectiveness of KMC}

Globally, the annual incidence of LBW infants constitutes a heavy burden on the health and social infrastructure of developing countries. Based on the complex nature of medical care for LBW infants, costly health infrastructure and highly skilled staff remain very essential for adequate neonatal care. In resource-limited settings, dearth of requisite health staff as well as ill-equipped neonatal care units constitute obstacles to implementing the $\mathrm{CMC} .{ }^{41} \mathrm{KMC}$ was thus initiated as the "natural" means of thermoregulation and neonatal care because it was meant to address the problems of shortage of incubators and the impact of maternal separation from newborn babies in neonatal care units.

As a cost-effective method for thermoregulation and care in LBW babies, KMC should be able to release scarce health resources consumed by the technology-based CMC. In the concept of cost-effectiveness, if these resources have a value in an alternative use, then the strategy can be credited with generating cost savings. The cost-effectiveness of KMC is thus assessed by comparing the incurred expenditure to the savings. When choices have to be made between KMC 
and $\mathrm{CMC}$ in this instance, the technique of incremental cost-effectiveness analysis has to be applied, where the cost of the strategy is represented in monetary terms and the benefits are measured in natural units common to both methods under consideration (KMC and CMC). ${ }^{42}$

Besides the published evidence on its safety and effectiveness, studies on its cost implications and the economic evaluation of the three components of KMC have also been documented. ${ }^{43-45}$

Ruiz et al performed a cost utility analysis on the results of a randomized control trial conducted in Bogotá, Colombia between 1993 and $1996 .{ }^{43}$ Hospital and ambulatory costs were estimated by micro-costing in a population of preterm LBW infants from a university hospital in Bogotá in 2011 and at a KMC clinic in the same period. Utility scores were assigned, 95\% CIs for the incremental cost-utility ratios (ICURs) were calculated, while one-way sensitivity analysis on price estimates for valuing costs was performed. The authors noted that the ICUR at 1 year of corrected age was US\$ 1,546 per extra quality-adjusted life year gained using the KMC method (95\% CI, - US\$ 7,963 to US\$ 4,910), prompting them to conclude that $\mathrm{KMC}$ was not only more effective and cost saving but could also be cost-effective in similar low- and middle-income settings, even though results from an economic analysis should not be extrapolated to different systems and communities. ${ }^{43}$

In another related study conducted in Nicaragua, Broughton et al examined the costs of implementing $\mathrm{KMC}$ in a referral hospital, including training, implementation, and ongoing operating costs and estimated the economic impact on the country's health system if KMC was implemented in other maternity hospitals in the same country. ${ }^{44}$ After receiving clinical training in $\mathrm{KMC}$, the implementation team trained their colleagues, defined guidelines for clinicians and education material for parents, and ensured adherence to the new guidelines. The study compared data on infant weight, medication use, formula consumption, incubator use, and admission for 6 months pre- and post-implementation. Cost data were obtained from accounting records of the implementers and health ministry formularies. The researchers found that neonates had post-implementation shorter durations of hospitalization by 4.64 days. In addition, the cost of the intervention stood at US\$23,133 but the money saved with shorter hospitalization, elimination of incubator use, and lower antibiotic and infant formula costs made up for this expenditure in 1-2 months. Extending KMC to 12 other facilities in Nicaragua was projected to save $\sim$ US\$ 166,000 (based on the referral hospital incubator-use estimate) or
US\$ 233,000 after 1 year (based on the more conservative incubator-use estimate). ${ }^{44}$

A multicenter, randomized controlled trial conducted for a year in three developing countries in Africa (Ethiopia), Asia (Indonesia), and South America (Mexico) evaluated the effectiveness, feasibility, acceptability, and cost of KMC when compared to $\mathrm{CMC} .{ }^{45} \mathrm{As}$ per cost, $\mathrm{KMC}$ was found to be less expensive than CMC in terms of salaries (US\$ 11,788 vs US\$ 29,888) and other running costs (US\$ 7,501 vs US\$ 9,876). The authors' report also confirmed the effectiveness and safety of hospital KMC when compared to CMC (for stabilized LBW infants), its feasibility in different settings, its acceptability to mothers of different cultural backgrounds, and its less expensive nature. ${ }^{45}$ Thus, for hospitals in low-income settings, KMC may represent an appropriate utilization of scarce resources, as well as an intervention to achieve an increase in prevalence and duration of exclusive breastfeeding with consequent benefits for health and growth. ${ }^{45}$

\section{Conclusion}

From the available evidence, $\mathrm{KMC}$ does have a comparative advantage over $\mathrm{CMC}$ especially with respect to improving neonatal survival, supporting exclusive breastfeeding, and promoting early discharge from the hospital. Although it was initially proposed for resource-limited settings to reduce the high neonatal mortality rates associated with preterm and LBW infants, KMC has now been endorsed by WHO for neonatal care in both high-income (developed) and low-income (developing) countries. In developed countries, there appears to be a gap in its implementation due to easy accessibility to incubators and other technology-based components of CMC. Much progress has, however, been made regarding its implementation in many developing countries where facility-based KMC has been institutionalized. Despite the proven cost-effectiveness of KMC in neonatal care, its global implementation is bedeviled with country-specific, multifaceted challenges. Nevertheless, many developing countries have initiated national policies to scale up KMC services in their domain. Given the major constraints to program implementation peculiar to these resource-limited countries, it has become imperative to boost caregiver confidence and experience using dedicated spaces in the hospital, as well as dedicated staff meant for adequate ambulatory follow-up and continuous health education. Therefore, capacity training for health professionals and provision of space infrastructure constitute the basic needs which could be funded by International Aid Agencies in order to scale up the program in these settings. 


\section{Disclosure}

The author reports no conflicts of interest in this work.

\section{References}

1. Charpak N, Ruiz JG. The kangaroo mother care method: from scientific evidence generated in Colombia to worldwide practice. J Clin Epidemiol. Epub 2016 Oct 17.

2. Çınara ND, Filiz TM. Neonatal thermoregulation. J Neonatal Nurs. 2006;12(2):69-74

3. Thomas K. Thermoregulation in neonates. Neonatal Netw. 1994;13(2): 15-22.

4. Gesta S, Tseng YH, Kahn CR. Developmental origin of fat: tracking obesity to its source. Cell. 2007;131(2):242-256.

5. Cinti S. The adipose organ. Prostaglandins Leukot Essent Fatty Acids. 2005;73(1):9-15.

6. Merenstein GB, Gardiner SI, editors. Handbook of Neonatal Intensive Care. 3rd ed. St Louis: Mosby; 1998.

7. Ekure EN, Iroha EO, Egri-Okwaji MTC, Ogedengbe OK. Perinatal mortality at the close of the 20th century in Lagos University Teaching Hospital. Niger J Paediatr. 2004;31:14-18.

8. Åhman E, Zupan J, editors. Neonatal and Perinatal Mortality: Country, Regional and Global Estimates 2004. Geneva: World Health Organization; 2007.

9. Park HK, Choi BS, Lee SJ, Son IA, Seol IJ, Lee HJ. Practical application of kangaroo mother care in preterm infants: clinical characteristics and safety of kangaroo mother care. J Perinat Med. 2014;42(2): 239-245.

10. Tuoni C, Scaramuzzo RT, Ghirri P, Boldrini A, Bartalena L. Kangaroo mother care: four years of experience in very low birth weight and preterm infants. Minerva Pediatr. 2012;64(4):377-383.

11. Bera A, Ghosh J, Singh A, Hazra A, Som T, Munian D. Effect of kangaroo mother care on vital physiological parameters of the low birth weight newborn. Indian J Community Med. 2014;39(4): 245-249.

12. Pattinson RC, Bergh AM, Malan AF, Prinsloo R. Does kangaroo mother care save lives? J Trop Pediatr. 2006;52(6):438-441.

13. Charpak N, Ruiz J, Zupan J, et al. Kangaroo mother care: 25 years after. Acta Paediatr. 2005;94(5):514-522.

14. Conde-Agudelo A, Diaz-Rossello JL, Belizán JM. Kangaroo mother care to reduce morbidity and mortality in low birthweight infants. Cochrane Database Syst Rev. 2000;4:CD002771.

15. Conde-Agudelo A, Belizán JM, Diaz-Rossello JL. Kangaroo mother care to reduce morbidity and mortality in low birthweight infants. Cochrane Database Syst Rev. 2011;3:CD002771.

16. Conde-Agudelo A, Díaz-Rossello JL. Kangaroo mother care to reduce morbidity and mortality in low birthweight infants. Cochrane Database Syst Rev. 2014;4:CD002771.

17. Conde-Agudelo A, Díaz-Rossello JL. Kangaroo mother care to reduce morbidity and mortality in low birthweight infants. Cochrane Database Syst Rev. 2016;8:CD002771.

18. Flacking R, Ewald U, Wallin L. Positive effect of kangaroo mother care on long-term breastfeeding in very preterm infants. J Obstet Gynecol Neonatal Nurs. 2011;40(2):190-197.

19. Bergh AM, Kerber K, Abwao S, et al. Implementing facility-based kangaroo mother care services: lessons from a multi-country study in Africa. BMC Health Serv Res. 2014;14:293.

20. Bergh AM, Manu R, Davy K, et al. Progress with the implementation of kangaroo mother care in four regions in Ghana. Ghana Med J. 2013;47(2):57-63.

21. Bergh AM, de Graft-Johnson J, Khadka N, et al. The three waves in implementation of facility-based kangaroo mother care: a multi-country case study from Asia. BMC Int Health Hum Rights. 2016;16:4.
22. Vesel L, Bergh AM, Kerber KJ, et al. Kangaroo mother care: a multicountry analysis of health system bottlenecks and potential solutions. BMC Pregnancy Childbirth. 2015;15(Suppl 2):S5.

23. Charpak N, Ruiz-Peláez J. Resistance to implementing kangaroo mother care in developing countries, and proposed solutions. Acta Paediatr. 2006;95(5):529-534.

24. Ludington-Hoe S, Lewis T, Morgan K, Cong X, Anderson L, Reese S. Breast and infant temperatures with twins during shared kangaroo care. J Obstet Gynecol Neonatal Nurs. 2006;35(2):223-231.

25. Tessier R, Cristo M, Velez S, et al. Kangaroo mother care and the bonding hypothesis. Pediatrics. 1998;102(2):e17-e33.

26. Moore ER, Bergman N, Anderson GC, Medley N. Early skin-to-skin contact for mothers and their healthy newborn infants. Cochrane Database Syst Rev. 2016;11:CD003519.

27. Ludington-Hoe S, Hosseini R, Torowicz D. Skin-to-skin contact (kangaroo care) analgesia for preterm infant heel stick. AACN Clin Issues. 2005;16(3):373-387.

28. Feldman R, Eidelman A, Sirota L, Weller A. Comparison of skin-toskin (kangaroo) and traditional care: parenting outcomes and preterm development. Pediatrics. 2002;110(1):16-26.

29. Penalva O, Schwartzman J. Descriptive study of the clinical and nutritional profile and follow-up of premature babies in a kangaroo mother care program. J Pediatr. 2006;82(1):33-39.

30. Johnston C, Stevens B, Pinelli J, et al. Kangaroo care is effective in diminishing pain response in preterm neonates. Arch Pediatr Adolesc Med. 2003;157(11):1084-1088.

31. Ellett M, Bleah D, Parris S. Feasibility of using kangaroo (skin-to-skin) care with colicky infants. Gastroenterol Nurs. 2004;27(1):9-15.

32. Zhang F, Liu S. Kangaroo mother care may help oral growth and development in premature infants. Fetal Pediatr Pathol. 2012;31:191-194.

33. Ndelema B, Van den Bergh R, Manzi M, et al. Low-tech, high impact: care for premature neonates in a district hospital in Burundi. A way forward to decrease neonatal mortality. BMC Res Notes. 2016;9:28.

34. Lincetto O, Nazir AI, Cattaneo A. Kangaroo mother care with limited resources. J Trop Pediatr. 2000;46(5):293-295.

35. Ramanathan K, Paul VK, Deorari AK, Taneja U, George G. Kangaroo mother care in very low birth weight infants. Indian J Pediatr. 2001;68(11):1019-1023.

36. Suman RP, Udani R, Nanavati R. Kangaroo mother care for low birth weight infants: a randomized controlled trial. Indian Pediatr. 2008;45(1):17-23.

37. Kadam S, Binoy S, Kanbur W, Mondkar JA, FernandezA. Feasibility of kangaroo mother care in Mumbai. Indian J Pediatr. 2005;72(1):35-38.

38. Worku B, Kassie A. Kangaroo mother care: a randomized controlled trial on effectiveness of early kangaroo mother care for the low birthweight infants in Addis Ababa, Ethiopia. J Trop Pediatr. 2005;51(2):93-97.

39. Lima G, Quintero-Romero S, Cattaneo A. Feasibility, acceptability and cost of kangaroo mother care in Recife, Brazil. Ann Trop Paediatr. 2000;20(1):22-26.

40. Charpak N, Ruiz-Peláez JG, Figueroa de CZ, Charpak Y. A randomized, controlled trial of kangaroo mother care: results of follow-up at 1 year of corrected age. Pediatrics. 2001;108(5):1072-1079.

41. Ruiz-Peláez JG, Charpak N, Cuervo LG. Kangaroo mother care, an example to follow from developing countries. BMJ. 2004;329:1179-1181.

42. Robinson R. Cost-effectiveness analysis. BMJ. 1993;307:793-795.

43. Ruiz JG, Charpak N, Castillo M, et al. Economic evaluation of kangaroo mother care: cost utility analysis of results from a randomized controlled trial conducted in Bogotá. J Clin Epidemiol. Epub 2016 Oct 28.

44. Broughton EI, Gomez I, Sanchez N, Vindell C. The cost-savings of implementing kangaroo mother care in Nicaragua. Rev Panam Salud Publica. 2013;34(3):176-182.

45. Cattaneo A, Davanzo R, Worku B, et al. Kangaroo mother care for low birthweight infants: a randomized controlled trial in different settings. Acta Paediatr. 1998;87(9):976-985. 


\section{Publish your work in this journal}

Research and Reports in Neonatology is an international, peer-reviewed, open access journal publishing original research, reports, editorials, reviews and commentaries on neonatal health. The manuscript management system is completely online and includes a very quick and fair peer-review system. Visit http://www.dovepress.com/testimonials.php to read real quotes from published authors.

Submit your manuscript here: https://www.dovepress.com/research-and-reports-in-neonatology-journal 